\title{
W135 invasive meningococcal infections imported from Sub-Saharan Africa to France, January to April 2012
}

I Parent du Châtelet (i.parent@invs.sante.fr) ${ }^{1}$, P Barboza $^{1}$, M K Taha $^{2}$

1. French Institute for Public Health Surveillance (Institut de veille sanitaire -InVS), Saint-Maurice, France

2. National Reference Center for Meningococci, Institut Pasteur, Paris France

Parent du Châtelet I, Barboza P, Taha MK. W135 invasive meningococcal infections imported from Sub-Saharan Africa to France, January to April 2012 . Euro Surveill. 2012;17(21): pii=20181. Available online: http://www.eurosurveillance.org/ViewArticle.aspx?Articleld=20181

From January to April 2012, 16 cases of W135 invasive meningococcal infection were reported in France. Of these, eight were linked to a recent travel history to Sub-Saharan Africa. These cases were reported in France concomitantly with the meningitis epidemic season in Sub-Saharan Africa. Considering the high number of travellers between France and West-African countries belonging to the so-called meningitis belt, the French recommendations for travellers stress the importance of vaccination before travelling to these countries.

In mid-February 2012, two W135 invasive meningococcal disease (IMD) cases were reported in two French regions (Pays de la Loire and Rhône-Alpes) in persons having recently returned from Senegal. The first case had arrived in France on 12 February and was hospitalised three days later. The second case arrived on 19 February and was hospitalised on the same day. No connection could be established between the two cases but they had both visited the same region in Senegal (near Mbour) and they were both working with non-governmental organisations (NGOs).

In France, the annual mean incidence of IMD varies between 0.9 and 1.5 cases per 100,000 population. Cases are mainly due to serogroup $B$ and $C$ meningococci ( $65 \%$ and $27 \%$ respectively for the last 10 years). Serogroup W135 is rare in France; sporadic cases were reported in the 1990 (less than five cases per year) and they mainly belonged to the clonal complexes ST-11 and ST-22 (French National Reference Centre for Meningococci (NRCM), unpublished data). However, this serogroup underwent a clonal expansion in France and other European countries in 2000, during the first reported multinational outbreak of serogroup W135 Neisseria meningitidis infections belonging to a particular clone of the ST-11 clonal complex. This outbreak started among pilgrims to Mecca and their contacts [1] and then affected Sub-Saharan countries (mainly Burkina Faso) $[2,3]$. Following a peak of incidence in 2002 with 42 reported cases, the incidence of $W_{135}$ (ST-11) IMD cases decreased in France and the W135 cases were most frequently due to isolates belonging to the clonal complex ST-22 representing in 2011 less than $3 \%$ of the cases with known serogroup (14/542) (NRCM, unpublished data).

\section{Investigation of W135}

\section{meningitis cases in 2012}

In 2012, the epidemic meningitis season, which coincides annually with the dry season between December and June, had already started in the so-called meningitis belt when the two W135 cases imported from Senegal were notified. Therefore we collected information regarding recent travel for all the W135 IMD cases that occurred in France since the beginning of the year.

Between 1 January and 1 April 2012, a total number of 16 IMD cases were notified in France. This is an important increase if compared to the previous five years when only four to eight W135 cases were reported each year during the first five months.

All 16 cases reported this year were laboratory-confirmed through isolation of $N$. meningitidis, positive PCR or detection of $N$. meningitidis antigens. The median age of cases was 45 years (range: 2 months to 89 years) and the $M: F$ ratio was 9:7. None of the 16 cases had been vaccinated with a tetravalent $A / C / Y /$ W135 meningococcal polysaccharide vaccine.

For eight of the 16 cases, a link to Sub-Saharan Africa was identified: two had returned from Senegal in February, one had arrived from Mali four days before the disease onset and one arrived from Senegal 15 days before the disease onset. The other four cases did not travel during their incubation period but a recent travel history was found for their close contacts: to Benin for one case, to Mali for two cases and to Senegal for one case with dates of return to France within the three weeks before the disease onset of the case. The purpose of travel was visiting friends and relatives for six cases or contacts and working for NGOs for two.

Considering an incubation period of 10 days, the dates of arrival in France and onset of the disease, three of 
the eight cases could be considered as imported. The remaining five cases may have been infected by asymptomatic contacts carrying an imported strain. However, strains were not investigated among contacts.

Among the eight cases, four cases presented with meningitis, two with pneumonia and septicaemia, one with arthritis, and one with pericarditis. No death was registered among the cases.

As of 24 May 2012, no other W135 IMD case has been notified since 1 April 2012.

All eight 'possibly import-related' W135 IMD cases were caused by the same strain, characterised at the NRCM in Paris by multilocus sequence typing, PorA variable regions (VR1 and VR2), penA and fet $A$ genes. The antigenic formula was $W_{135}: 2 a: P_{1-5}, 2$, the genetic typing showed porA $V R_{1}=5, V R 2=2$, fet $A=F_{1}-1$, pen $A=1$, and the strains were ST-11.

\section{Conclusions}

The increase of serogroup W135 meningococcal disease incidence in France in early 2012 was concomitant to the meningitis epidemic season in Sub-Saharan Africa. From 1 January to 15 April 2012, almost 15,000 meningitis cases have been reported in West Africa to the World Health Organization. In some countries (e.g. Burkina Faso, Côte d'Ivoire, Ghana, Mali, Niger) the serogroup $W_{135}$ was predominant among cases for which $N$. meningitidis has been identified whereas serogroup A was predominant in other countries like Chad [4]. Serogroup $W_{135}$ has increased in Niger in 2010 [5]. No laboratory results were available from Senegal.

The French NRCM typed eight isolates from Côte d'Ivoire. These bacteria were isolated in February 2012 in three different districts of the country. All the isolates from France and Côte d'Ivoire shared the same tested markers (porA VR1=5, VR2 $=2$, fet $A=F_{1}-1$, pen $A=1, S T-11$ ) (unpublished data). Eight other cases of W135 cases were also isolated in France during the same period but they reported no travel history during the previous three months. All these isolates also showed different markers (unpublished data).

Further investigations are required including typing isolates from other countries within the meningitis belt and comparing results with isolates from the previous years in order to help understanding this recent re-emergence of $\mathrm{W}_{135} / \mathrm{ST}-11$ isolates. A long-lasting establishment of this serogroup in sub-Saharan African countries may prompt re-considering the vaccination strategies in the belt upon the introduction of the conjugate vaccine against serogroup A [6].

In the meantime, specific surveillance should be enhanced in Europe and recommendations for travellers who have contacts with population in high-incidence countries should be updated taking into account that non-pilgrimage-related travel is rarely associated with transmission and that the purposes of travel described above for the cases themselves or their asymptomatic contacts were visits to family members and friends or work for NGOs [7]. Since there are frequent travellers between France and West-African countries belonging to the meningitis belt, the French recommendations for travellers insist on the importance of vaccination with a quadrivalent $A / C / Y / W 135$ vaccine (preferably a conjugate vaccine) when travelling to these countries [8].

\section{References}

1. Taha MK, Achtman M, Alonso JM, Greenwood B, Ramsay M, Fox A, et al. Serogroup W135 meningococcal disease in Haji pilgrims. Lancet 2000;356(9248):2159.

2. Aguilera JF, Perrocheau A, Meffre C, Hahné S; W135 Working Group. Outbreak of serogroup W135 meningococcal disease after the Hajj pilgrimage, Europe, 2000. Emerg Infect Dis. 2002;8(8):761-7.

3. Mayer LW, Reeves MW, Al-Hamdan N, Sacchi CT, Taha MK, Ajello GW, et al. Outbreak of W135 meningococcal disease in 2000: not emergence of a new W135 strain but clonal expansion within the electophoretic type-37 complex. J Infect Dis. 2002;185(11):1596-605.

4. World Health Organization (WHO) Regional Office for Africa. Meningitis weekly bulletin. Weekly feedback bulletin on cerebrospinal meningitis. Burkina Faso: WHO: Apr 2012. Available from: http://www.meningvax.com/files/ BulletinMeningite2012_S15.pdf

5. Collard JM, Maman Z, Yacouba H, Djibo S, Nicolas P, Jusot JF, et al. Increase in Neisseria meningitidis serogroup W135, Niger, 2010. Emerg Infect Dis. 2010;16(9):1496-8.

6. Kristiansen PA, Ouédraogo AS, Sanou I, Ky Ba A, Ouédraogo $C D$, Sangaré $L$, et al. Laboratory quality control in a multicentre meningococcal carriage study in Burkina Faso. Trans R Soc Trop Med Hyg. 2012;106(5):289-97.

7. Koch S, Steffen R. Meningococcal Disease in Travelers: Vaccination Recommendations. J Travel Med.1994.1(1): 4-7.

8. Comité des maladies liées aux voyages et d'importation (CMVIHCSP). Health recommendations for travellers, 2011 (for health professionals). BEH. 2011;18-19: 205-8. Available from: http:// www.invs.sante.fr/beh/2011/18_19/beh_18_19.pdf 